\title{
The Effect of Low Level Laser Therapy and Acupressure in Controlling Gag Reflex in Children Undergoing Dental Procedures
}

\author{
Paloni Koticha ${ }^{1}$, Farhin Katge ${ }^{2}$, Vamsikrishna Chimata ${ }^{3}$, Manohar Poojari ${ }^{3}$, \\ Shilpa Shetty ${ }^{3}$ \\ ${ }^{1}$ Senior Lecturer, ${ }^{2}$ Professor and Head of Department, ${ }^{3}$ Reader, \\ Department of Paedodontics and Preventive Dentistry, Terna Dental College, Nerul, Navi Mumbai \\ Corresponding Author: Farhin Katge
}

\begin{abstract}
Background: Impression making can be challenging in children due to the exaggerated gag reflex. It not only hinders the basic dental examination procedure but also delivery of the treatment procedure becomes more challenging.

Aim: Comparative evaluation of Low Level Laser Therapy (LLLT) and in combination with acupressure in controlling gag reflex in children undergoing impression making procedure.

Experimental Procedure: A total of 90 children $(10.1 \pm 2.6)$ years were divided into three groups depending upon the strategies used. Group A received LLLT whereas group B received LLLT with acupressure and group C was placebo group. Each patient underwent two impressions to record GSI and GPI scores for all groups.

Results: Mean values of GSI and GPI scores for each group were calculated at three different stages. The mean values revealed least gagging with LLLT as compared to LLLT with acupressure and placebo group at stage 1 (p$0.001)$ and stage 3 (p-0.000) which was statistically significant. Mean values of GPI score was less than GSI score at all three stages for all the groups except for stage 1 in group C, which was not statistically significant.

Conclusion: LLLT and in combination with acupressure was effective in controlling gag reflex in comparison to acupressure in children undergoing impression making procedure.
\end{abstract}

Keywords: Acupuncture, acupressure, acupoints, gag reflex, impression making

\section{INTRODUCTION}

Impression making can be clinically challenging in pediatric patients due to child's apprehension and fear for dental procedure. In addition gag reflex and hyperactive tongue further hinders the usual treatment procedures. ${ }^{[1]}$ Gag reflex also called as pharyngeal reflex can be defined as a physiological mechanism to protect upper respiratory tract from the aspiration of foreign bodies. [2] Apart from its physiological nature, it can also be acquired by various stimuli like visual, olfactory, toxic or chemicals diffused through blood. [3] Presence of trigger zones like palatoglossal and palatopharyngeal folds along with the base of tongue, palate, uvula and posterior pharyngeal wall make the oral cavity sensitive to such stimuli. ${ }^{[4]}$

When gag reflex is mild in nature it can be managed with slight modifications in treatment procedure like application of local anaesthetic spray on the trigger zones. [5] However, when severity of this reflex increases various other strategies have been proposed. These range from use of behaviour modification techniques to use of acupuncture. [6] Low level laser therapy (LLLT) and acupressure are variants of acupuncture that utilises similar specific points in order to maintain general wellbeing. ${ }^{[7]}$ As a traditional Chinese medicine, acupuncture elucidates the balance between the positive and negative 
forces of the universe and thus forms a key in understanding any ailment. [8] Acupressure is a non-invasive technique that relies on the concept of meridian. ${ }^{[8]}$ Meridians act as a pathway through which life energy flows also called as 'Qi'. ${ }^{[8]}$ The theory involves stimulation of acupoints that lie on these meridians in the body to increase bioenergy, consequently reducing the symptoms. ${ }^{[7]}$ In context to control of gag reflex various acupoints are suggested like Pericardium 6 (PC-6) and Conception Vessel 24 (CV-24). ${ }^{[10]}$ These acupoints can be stimulated by hand, foot or use of acupressure band. ${ }^{[9]}$ On the other hand, LLLT also called as laser acupuncture or biostimulation therapy is based on use of light with single wavelength. ${ }^{[11]}$ Low level lasers used for this therapy do not increase the temperature in the tissues rather induce photobiostimulation within the tissues. ${ }^{[12]}$ Thus this study was conducted to evaluate the effect of LLLT and in combination with acupressure in controlling gag reflex in children undergoing impression making procedure. The null hypothesis proposed was that there is no difference in effect of low level laser therapy or its combination with acupressure in controlling gag reflex in patients requiring upper alginate impression.

\section{MATERIAL AND METHODS}

The study was conducted in the Department of Pediatric and Preventive Dentistry. The study protocol was approved from the institutional review board of ethics (TDC/IRB-EC/126/2016). The study was conducted in accordance with the 1964 Helsinki declaration.

\section{Subject Enrollment}

Sample size calculation was done using $\mathrm{G}^{*} \mathrm{P}$ (version 3.1.9.2, Heirnrich Heine University, Germany). Effect size was taken as 0.5 , the alpha probability was 0.05 . Total sample size was calculated to be 90 . Parents were explained about the purpose of study and written consent was taken for the same. Presence of gag reflex for these volunteers was assessed by stimulating posterior hard palate with the back of disposable mirror. ${ }^{[13]}$ Children selected for the present study had to undergo two maxillary impression making procedures. First impression was made to assess the severity of gag reflex while the second impression was made using the two different treatment modalities. Randomization was done using sequentially sealed opaque envelopes with 30 patients in each group.

Group A $(\mathbf{n}=\mathbf{3 0})$ : Laser stimulation at CV24

Group B $(\mathbf{n = 3 0})$ : Laser stimulation at CV24 and wrist band at PC-6

Group C $(\mathbf{n}=\mathbf{3 0})$ : Laser stimulation at placebo point at PC-3

\section{Inclusion and Exclusion Criteria}

Children (38 girls, 52 boys) aged between 10 to 13 years were selected for the study. Those children who displayed moderate (Grade 3) to severe (Grade 4) gag reflex according to gag severity index (GSI) during dental examination and requiring upper arch impression for diagnostic purpose were included in the study. On the other hand, children who were uncooperative as well as those with special health care needs were excluded from the study.

\section{Description of the Acupoints}

Acupoints CV-24 and PC-6 were selected for the present study. CV-24 is located at the mentolabial sulcus. (Figureureure.1) ${ }^{[14]}$ PC-6 is located on the anterior forearm, 2 cun proximal to the transverse wrist crease, between the tendons of palmaris longus and flexor carpi radialis. In acupuncture cun is a measurement relative to the patient's body that is used to locate acupoints. 1 cun is equal to patient`s thumb. ${ }^{[15]}$ The acupoint is located with the measurement equivalent to the breadth of patient's three fingers on the anterior surface of the wrist, above the distal skin crease on the wrist joint (Figure 2). Here, 1 cun is equal to patient's thumb. ${ }^{[14]}$ In group $\mathrm{C}$ the placebo point $\mathrm{PC} 3$ (Pericardium 3) was selected. It is located 
on the forearm measuring 6 cuns from the wrist of the patient. (Figure 3) ${ }^{[16]}$

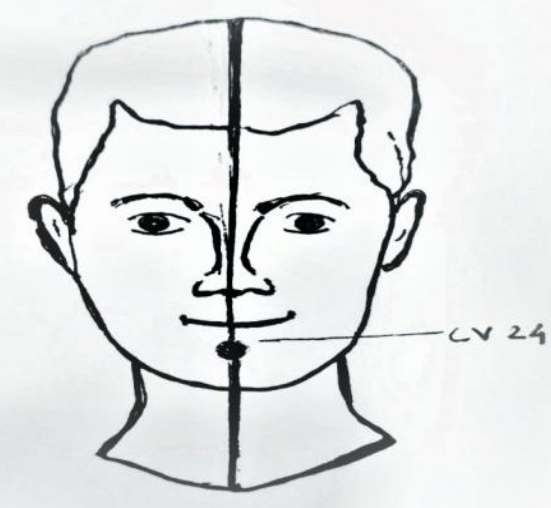

Figure 1: Diagrammatic representation of CV-24

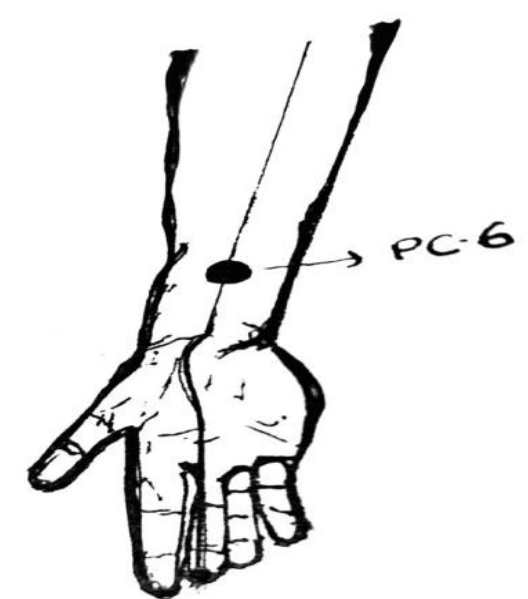

Figure 2: Diagrammatic representation of PC-6

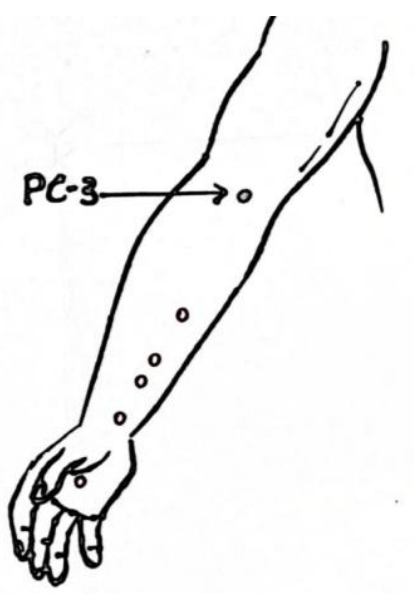

Figure 3: Diagrammatic representation of PC-3

\section{Scoring Criteria}

Criterion proposed by Dickinson and Fiske was used to assess gag reflex in the study. ${ }^{[17]}$ The criteria comprises of gag severity index (GSI) and gag preventive index (GPI). GSI measures the reactivity of the patient to gag causing stimulus which is rated on a scale from mild (grade 1) to very severe (grade 5). On the other hand, GPI assessed the efficacy of the treatment employed for the control of gag reflex. It ranges from obtunded gag reflex with successful treatment (Grade 1) to severe gag reflex where treatment is not possible (Grade 5). The scoring was done at three stages of impression making.

Stage I: Evaluation of the patient's ability to tolerate an empty tray (placed in the mouth).

Stage II: Evaluation of the patient's ability to tolerate a tray loaded with alginate (placed in the mouth).

Stage III: Evaluation of the patient's ability to tolerate the loaded impression tray until the alginate set.

\section{Treatment Procedure}

All impression making procedures were carried out using sterilised perforated stock trays. Proper tray selection was carried for every child in each group. The impression was made using Alginate (Tropicalgin, Zhermack, Italy). The material was manipulated for $1 \mathrm{~min} 50$ seconds according to the manufacturer's instructions. The first maxillary impression was made for all children in three groups to assess the severity of gag reflex using GSI. Following this a second impression was made for children in each group to assess GPI. Since the study was a crossover design a time gap of 30 minutes was allotted between two impression making procedures. The children were allocated into the following groups:

\section{Treatment Procedure}

All impression making procedures were carried out using sterilised perforated stock trays. Proper tray selection was carried for every child in each group. The impression was made using Alginate (Tropicalgin, Zhermack, Italy). The material was manipulated for $1 \mathrm{~min} 50$ seconds according to the manufacturer's 
instructions. The first maxillary impression was made for all children in three groups to assess the severity of gag reflex using GSI. Following this a second impression was made for children in each group to assess GPI. Since the study was a crossover design a time gap of 30 minutes was allotted between two impression making procedures. The children were allocated into the following groups:

Group A: Children in this group underwent laser therapy (Elexxion AG- Dental Laser, Germany) with power output $0.5 \mathrm{~mW}$, wavelength $810 \mathrm{~nm}$ on defocused energy mode stimulation at CV-24 during impression making procedure. Marking of the acupoint was done before starting the procedure. Activation of the acupoint was carried out by focusing the laser with a spot size of 1 inch in diameter for 1 minute while keeping the tip of the laser 3-4 mm away from the spot. (Figureureure.4) The energy fluence of the laser was calculated according to the following formula: ${ }^{[18]}$ $\mathrm{H}=\mathrm{Q} / \mathrm{a}$

Where, $\mathrm{H}$; fluence energy density, Q; radiant energy and a: area of spot size $\mathrm{r}^{2}$

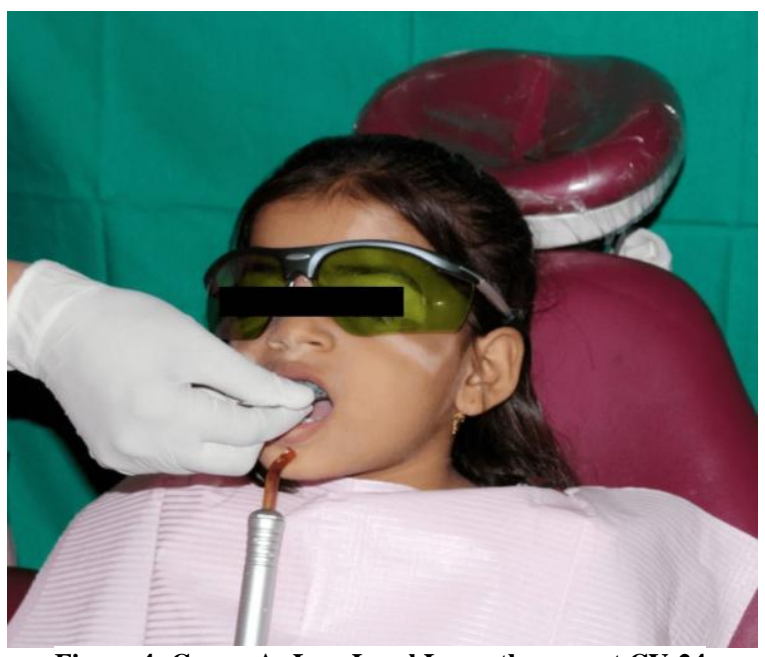

Figure 4: Group A- Low Level Laser therapy at CV-24

Group B: In this group children underwent laser (Elexxion AG- Dental Laser, Germany) stimulation at CV-24 along with acupressure at PC-6 simultaneously. After locating the acupoint PC-6 patients were made to wear a magnetic wrist band (SeaBand International, Newport, RI UK) which consisted of a button for application of pressure at this acupoint. This wrist band was worn by the child for 20 minutes prior to the impression making procedure. Acupoint CV-24 was then stimulated with laser similar to group A along with the magnetic wrist band in place during impression making. (Figure 5)

Group C: Children in this group underwent laser stimulation at placebo point (PC-3). After marking the acupoint, activation was done using low level laser while impression was being made. The mode and spot size being similar to that in group A (Figure 6)

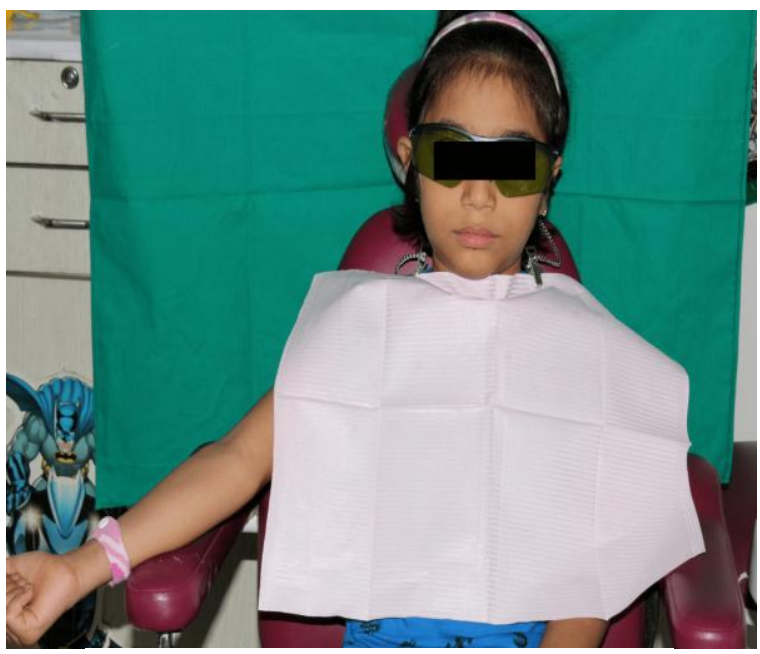

Figure 5: Use of Seaband for acupressure at PC-6

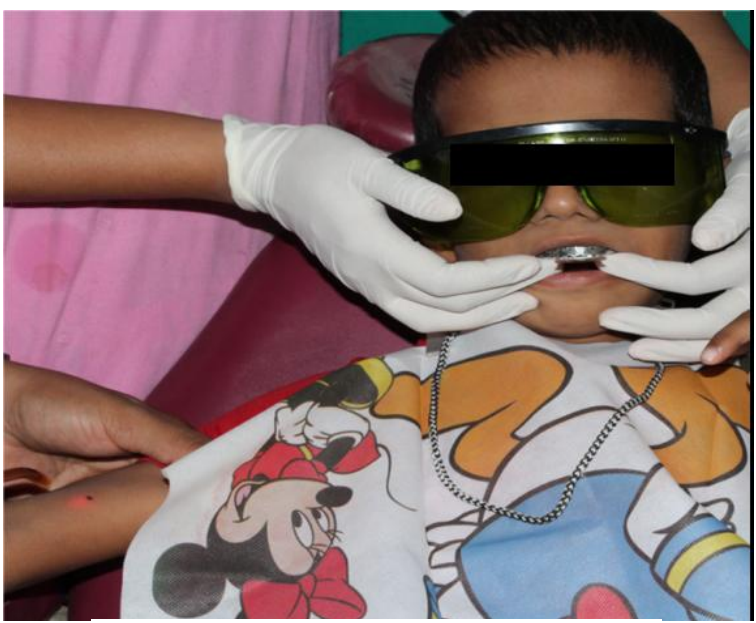

Figure 6: LLLT stimulation at placebo acupoint

\section{Statistical analysis}

Statistical analysis was done using the SPSS (SPSS Inc., Chicago IL) version 25 software. GSI and GPI scores between the groups were evaluated using one way ANOVA test. Comparison between the stages was done using general linear model. Comparison within the groups was done 
Paloni Koticha et.al. The effect of Low Level Laser Therapy and acupressure in controlling gag reflex in children undergoing dental procedures

using paired $\mathrm{t}$ test. The statistical significance level was set at $\mathrm{p}<0.05$.

\section{RESULTS}

Patients (38 girls, 52 boys) in the mean age group of $10.1 \pm 2.6$ years were included in the present study. Data obtained was entered in Microsoft Excel 2013. Frequency and descriptive analysis were done by using statistical package for social sciences software (SPSS) (v.21.0). Statistical analysis was performed using One Way ANOVA followed by Post hoc Tukey's test at $95 \%$ confidence intervals. Mean values of GSI and GPI scores for each group were calculated at three different stages. The mean values revealed least gagging with LLLT as compared to LLLT with acupressure and placebo group at stage 1 (p-0.001) and stage 3 (p-0.000) which was statistically significant. Mean values of GPI score was less than GSI score at all three stages for all the groups except for stage 1 in group $\mathrm{C}$, which was not statistically significant.

Table 1: Intergroup comparison at stage 1, 2, 3 before laser acupuncture and acupressure in all the groups (Gag severity index)

\begin{tabular}{|c|c|c|c|c|c|c|}
\hline & \multicolumn{2}{|c|}{ Stage 1 } & \multicolumn{2}{c|}{ Stage 2 } & \multicolumn{2}{c|}{ Stage 3 } \\
\hline & Mean & SD & Mean & SD & Mean & SD \\
\hline Group A & 2.70 & 0.952 & 2.83 & 1.053 & 2.93 & 0.691 \\
\hline Group B & 2.97 & 0.765 & 2.70 & 0.596 & 2.97 & 0.718 \\
\hline Group C & 2.90 & 0.481 & 2.67 & 0.547 & 2.90 & 0.662 \\
\hline P Value & \multicolumn{2}{|c|}{$\mathbf{0 . 3 7 0}$} & \multicolumn{2}{c|}{$\mathbf{0 . 6 7 4}$} & \multicolumn{2}{c|}{$\mathbf{0 . 9 3 3}$} \\
\hline
\end{tabular}

Table 2: Intergroup comparison at stage 1, 2, 3 during laser acupuncture and acupressure in all the three groups (Gag preventive Index)

\begin{tabular}{|c|c|c|c|c|c|c|}
\hline & \multicolumn{2}{|c|}{ Stage 1} & \multicolumn{2}{|c|}{ Stage 2} & \multicolumn{2}{|c|}{ Stage 3} \\
\hline & Mean & SD & Mean & SD & Mean & SD \\
\hline Group A & 2.40 & 0.675 & 2.50 & 0.861 & 2.13 & 0.681 \\
\hline Group B & 2.90 & 0.481 & 2.67 & 0.547 & 2.77 & 0.728 \\
\hline Group C & 3.03 & 0.765 & 2.43 & 0.722 & 2.90 & 0.662 \\
\hline P Value & \multicolumn{2}{|c|}{0.001} & \multicolumn{2}{|c|}{0.440} & \multicolumn{2}{|c|}{0.000} \\
\hline
\end{tabular}

Table 3: Results of GSI and GPI scores in group A

\begin{tabular}{|l|c|c|c|c|c|}
\hline \multirow{2}{*}{ Stages } & \multicolumn{2}{c|}{ GAG SEVERITYINDEX } & \multicolumn{2}{c|}{ GAG PREVENTIONINDEX } & \multirow{2}{*}{ P Value } \\
\cline { 2 - 5 } & Mean & SD & Mean & SD & \\
\hline Stage 1 (EMPTYTRAY) & 2.7000 & .95231 & 2.4000 & .67466 & 0.693 \\
\hline Stage 2 (LOADED TRAY) & 2.8333 & 1.05318 & 2.5000 & .86103 & 0.765 \\
\hline Stage 3 (ALGINATE SET) & 2.9333 & .69149 & 2.1333 & .68145 & 0.778 \\
\hline
\end{tabular}

Table 4: Results of GSI and GPI scores in group B

Table 4: Results of GSI and GPI scores in group B
\begin{tabular}{|l|c|c|c|c|c|}
\hline Stages & GAG SEVERITY INDEX & GAG PREVENTIVEINDEX & P Value \\
\hline & Mean & SD & Mean & SD & \\
\hline Stage 1 (EMPTY TRAY) & 2.9000 & 0.48066 & 3.0333 & .76489 & 0.961 \\
\hline Stage 2 (LOADEDTRAY) & 2.6667 & 0.54667 & 2.4333 & 0.72793 & 0.762 \\
\hline Stage 3 (ALGINATESET) & 2.7667 & 0.72793 & 2.9000 & 0.66176 & 0.306 \\
\hline
\end{tabular}

Table 5: GSI and GPI SCORES for group C

\begin{tabular}{|l|c|c|c|c|c|}
\hline \multirow{2}{*}{ Stages } & \multicolumn{2}{|c|}{ GAG SEVERITYINDEX } & GAG PREVENTION INDEX & \multirow{2}{*}{ P Value } \\
\cline { 2 - 5 } & Mean & SD & Mean & SD & \\
\hline Stage 1(EMPTYTRAY) & 2.9667 & 0.76489 & 2.9000 & 0.48066 & 0.657 \\
\hline Stage 2 (LOADEDTRAY) & 2.7000 & 0.59596 & 2.6667 & 0.54667 & 0.261 \\
\hline Stage 3 (ALGINATESET) & 2.9667 & .71840 & 2.7667 & 0.72793 & 0.540 \\
\hline
\end{tabular}

\section{DISCUSSION}

Gag reflex is a complex physiological defense mechanism to protect the upper respiratory tract. It can range from mild choking to violent retching that may or may not precede vomiting as suggested by Faigenblum. ${ }^{[19]}$ Considering the origin of gag reflex, it can be categorized into psychogenic and somatic type. Psychogenic response can either be correlated with negative dental experience or may not have any direct stimulus. On the other hand, somatic type of gag reflex can be induced by intraoral stimuli like placement of mouth mirror in intraoral examination. ${ }^{[20]}$ On stimulation afferent impulses are sent to chemoreceptor trigger zone (CTZ) in medulla oblongata. In response efferent impulses are released with spasmodic movements associated with the reflex. Moreover, close vicinity of CTZ to vomiting, salivating and cardiac centers further explains the associated responses with the centers. ${ }^{[21]}$ The severity of this reflex differs in every child. Occurrence of exaggerated gag reflex hinders the basic protocols of diagnosis and treatment that include use of impression trays or dental 
films. Extensive research on management of gag reflex is continued over years. Many behavior management techniques have been used to reduce its incidence by conversing, distracting or desensitizing the child. [6] Additional treatment modalities like relaxation techniques, pharmacological agents or acupuncture have also been used. [6] Acupuncture, traditional Chinese medicine has many modifications that can be used to treat any ailment like mild to moderate gag reflex. Of which LLLT and acupressure are considered to be very effective for its control based on the vast scientific literature available. ${ }^{[22,23]}$ In current study, red light $(810 \mathrm{~nm})$ was used as low level laser due to its property of less adsorption and more transmission. Acupressure was carried out using magnetic wrist band (Sea-Band International, Newport, RI UK) that consist of a button that applies pressure on the acupoint (PC-6) to alter the symptoms of gag reflex. Role of these modalities in control of gag reflex is attributed to release of various neurotransmitters like substance $P$, serotonin and cholecystokinin. Acupressure is an alternative technique which involves application of simple sustained pressure on an acupoint for two minutes using small circulatory movement. Because of the sustained pressure the afferent nerves type 1 and type 2 are activated which then send stimulus to the spinal cord. This results in release of endorphins that block the chemoreceptor trigger zones. ${ }^{[24]}$ The role of LLLT in controlling gag reflex relies on activation of the indicated acupoints which stimulates the specific regions of the brain leading to release of endorphins and encephalins. ${ }^{[25]}$ Considering the difficulty with traditional acupuncture, its variants LLLT and acupressure allows painless stimulation of the acupoints due to which they were considered for the present study. Moreover, the acupoints CV-24 and PC-6 were selected in the present study for their increased efficacy in controlling gag reflex. ${ }^{[20,21]}$ Therefore, the present study investigated the effect of laser stimulation of the acupoint CV- 24 and the combination of laser stimulation and acupressure at CV-24 and PC-6 respectively. This efficacy was assessed using the GSI and GPI index suggested by Dickson and Fiske. ${ }^{[17]}$ On tabulation of data of the current study showed mean values with least gagging for LLLT as compared to LLLT with acupressure and placebo group at stage 1 and stage 3 which was statistically significant suggesting the effective role of CV-24 in gag reflex. Various studies carried out by Elbay et al and Rosted et al gave comparable results showing similar results with CV-24 in control of gag reflex..$^{[18,25]}$ Moreover, none of the complications mentioned in the literature were observed in the present study. However, Mean values of GPI score was less than GSI score at all three stages for all the groups except for stage 1 in group $\mathrm{C}$, which was not statistically significant. The oddity of the mean values in the present study can be attributed two factors. Firstly, sudden untoward movement of the child while LLLT at CV-24. ${ }^{[13]}$ Secondly, inadequate stimulation of PC-6 due to hormonal imbalance in the child due to the nature and built of the child as suggested by Lu et al. ${ }^{[9]}$ Similar findings in a study conducted by Pol et al also showed the synergistic effect of both the acupoints in control of gag reflex. [21] Moreover, there was no significant difference was found in terms of GSI scores in any of the groups which can be attributed to the child`s perception of fear and anxiety towards the impression procedure. ${ }^{[26]}$ Therefore the current study suggest reduced gag reflex in children with either LLLT or combination of LLLT with acupressure. However, further studies are required with larger sample size due to the paucity in literature for effectiveness of LLLT and acupressure in children undergoing dental treatment.

\section{CONCLUSION}

Variations in treatment protocol along with use of different strategies to control gag reflex in children not only 
enhances patient acceptance but also the treatment outcome. The technique used in the present study LLLT alone and in combination with acupressure was effective in controlling gag reflex children undergoing impression making procedure. Moreover, owing to its safe and reliable outcome its use can be considered in children.

\section{Acknowledgement: None}

\section{Support: Nil}

\section{Conflict of Interest: Nil}

\section{Permissions: Nil}

Ethical Approval: Ethical clearance was obtained from the institutional review board of ethics, Navi Mumbai (TDC/IRBEC/120/2016).

\section{REFERENCES}

1. Kumar S, Satheesh P, Savadi RC. Gagging. N Y State Dent J. 2011; 77(4):227.

2. Bassi GS, Humphris GM, Longman P. The etiology and management of gagging: A review of the literature. Journal of Prosthetic Dentistry 2004; 91:459- 67.

3. Goyal G. Gag reflex: Causes and management. International Journal of Medical and Dental Research 2014; 1 (3): 163-166.

4. Meeker HG, Magalee R. The conservative management of the gag reflex in full denture patients. N Y State Dent J 1986; 52:11-4.

5. Eachempati P, Kumbargere Nagraj S, Kiran Kumar Krishanappa S, George RP, Soe HHK, Karanth L. Management of gag reflex for patients undergoing dental treatment. Cochrane Database Syst Rev. 2019; 2019(11):CD011116.

6. Hsu DT. Acupuncture: A Review. Reg Anesth Pain Med 1996; 21:361-70.

7. Sierpena V,Frenkel M. Acupuncture: A clinical review. South Med J 2005; 98 (3): 330-7

8. Cox, L., Brindley, J. Exploring alternative methods of gag reflex control Part 1: Acupressure. BDJ Team 4, 17059 (2017). https://doi.org/10.1038/bdjteam.2017.59
9. $\mathrm{Lu} \mathrm{DP}, \mathrm{Lu} \mathrm{GP}$, Reed JF 3rd. Acupuncture/acupressure to treat gagging dental patients: a clinical study of antigagging effects. Gen Dent 2000;48:446-52

10. Chon TY, Mallory MJ, Yang J, Bublitz SE, Do A, Dorsher PT. Laser Acupuncture: A Concise Review. Med Acupunct. 2019; 31 (3):164-8.

11. Anand MV, Rai R, Bettie NF, Ramachandiran H, Solomon, Praveena S. Acupuncture-An effective tool in the management of gag reflex. J Pharm Bioallied Sci. 2015 Aug; 7(Suppl 2):S677-9.

12. Sun G, Tunér J. Low-level laser therapy in dentistry. Dental Clinics of North America. 2004 Oct; 48(4):1061-76.

13. Goel H, Mathur S, Sandhu M, Jhingan P, Sachdev V. Effect of Low-level LASER Therapy on P6 Acupoint to Control Gag Reflex in Children: A Clinical Trial. J Acupunct Meridian Stud. 2017; 10(5):31723.

14. Usichenko TI, Müller-Kozarez I, Knigge S, Busch R, Busch M. Acupuncture for Relief of Gag Reflex in Patients Undergoing Transoesophageal Echocardiography-A Protocol for a Randomized PlaceboControlled Trial. Medicines (Basel). 2020; 7(4):17.

15. Yin CS, Park HJ, Seo JC, Lim S, Koh HG. Evaluation of the cun measurement system of acupuncture point location. Am J Chin Med. 2005; 33(5):729-35.

16. Kim J, Kang DI. Positioning Standardized Acupuncture Points on the Whole Body Based on X-Ray Computed Tomography Images. Med Acupunct 2014; 26(1):40-9.

17. Dickinson C M, Fiske J. A review of gagging problems in dentistry: 2. Clinical assessment and management. Dent Update. 2005; 32: 74-80.

18. Elbay M, Tak Ö, Şermet Elbay Ü, Kaya C, Eryılmaz K. The use of low-level laser therapy for controlling the gag reflex in children during intraoral radiography. Lasers Med Sci. 2016 Feb; 31(2):355-61.

19. Kaira L, Dabral E,Kukreja H. Gagging: A review. NUJHS. 2014; 4(1):149-55.

20. Singh S, Ali FM, Nazirkar G, Dole VK, Gaikwad B. Gag- Etiology and its skillful management- A review. J. Evolution Med. Dent. Sci. 2013; 2(10):1509-16.

21. Becker DE. Nausea, vomiting, and hiccups: a review of mechanisms and treatment. Anesth Prog. 2010; 57(4):150-7. 
Paloni Koticha et.al. The effect of Low Level Laser Therapy and acupressure in controlling gag reflex in children undergoing dental procedures

22. Pol S, Katge F, Chimata V, Poojari M, Balgi P, Shetty A. The Role of Laser Stimulation (Electro-acupuncture) in Alginate Impression Making for Patients with a Gag Reflex. Dent Asso Thai.2017;67(4):288-95.

23. Sari E, Sari T. The role of acupuncture in the treatment of orthodontic patients with a gagging reflex: a pilot study. Br Dent J. 2010; 208(10):E19.

24. Steele NM, French J, Gatherer-Boyles J, Newman S, Leclaire S. Effect of acupressure by Sea-Bands on nausea and vomiting of pregnancy. J Obstet Gynecol Neonatal Nurs. 2001; 30(1):61-70.

25. Rosted P, Bundgaard M, Fiske J, Pedersen AM. The use of acupuncture in controlling the gag reflex in patients requiring an upper alginate impression: an audit. Br Dent J. 2006 Dec 9; 201(11):721-5.

26. Ramsay D .Problematic gagging. Principles of treatment. J Am Dent Asso.1987; 114: 178-83.

How to cite this article: Koticha P, Katge F, Chimata V et.al. The effect of Low Level Laser Therapy and acupressure in controlling gag reflex in children undergoing dental procedures. International Journal of Science \& Healthcare Research. 2021; 6(2): 1-8. DOI: https://doi.org/ 10.52403/ijshr.20210401 